LA PLATA-TH 96/12

hep-th/9608192

\title{
Massless fermions in a bag at finite density and temperature
}

\author{
M. De Francia, H. Falomir \\ Departamento de Física, Facultad de Ciencias Exactas, Universidad Nacional de La Plata, \\ C.C. 67, 1900 La Plata, Argentina \\ M. Loewe \\ Facultad de Física, Pontificia Universidad Católica de Chile, \\ Casilla 306, Santiago 22, Chile
}

(June 28, 2021)

\begin{abstract}
We introduce the chemical potential in a system of massless fermions in a bag by impossing boundary conditions in the Euclidean time direction. We express the fermionic mean number in terms of a functional trace involving the Green's function of the boundary value problem, which we study analytically. Numerical evaluations are made, and an application to a simple hadron model is discussed.

PACS number(s): 03.65.Db, 11.10.Wx, 12.39.Ba, 12.38.Mh
\end{abstract}

\section{INTRODUCTION}

Functional determinants of elliptic differential operators have wide application in describing one-loop effects in Quantum and Statistical Physics. When a bounded manifold in the Euclidean space-time is considered, boundary conditions have a relevant influence on the behavior of such determinants.

In previous papers, we studied the relationship between functional determinants of elliptic boundary value problems and the corresponding Green's functions 1 1 3. This allowed for the description of confined fields at finite temperature.

In the framework of this functional techniques, we studied, in a simplified scenario in two dimensions, the Gibbs free energy of confined massless fermions [4]. The chemical potential $\mu$ was introduced through suitable boundary conditions impossed on the Dirac operator in the Euclidean time direction.

The present paper is an extension of that work: Here we consider a similar calculation for a four dimensional M.I.T. bag model. In this effective model of hadrons, a massless fermionic field is confined into a static sphere of radius $R$ and subject to local spatial boundary conditions. This problem has been considered previously [5] through an asymptotic expansion of the energy density, for large values of the bag radius. As we will show, the knowledge of the complete Green's function for this boundary value problem allows us to describe the mean fermionic number and the Gibbs free energy even at small radius, thus improving previous approaches.

The behavior of hadronic matter at finite temperature and density has received considerable attention [6] (see also (7] and references therein) during the last years. The main motivation behind this effort is an attempt to understand properly the generally accepted possibility of a deconfining phase transition from hadronic matter to the quark-gluon plasma [8], with applications to relativistic heavy ion collisions and to the early Universe.

Different techniques have been used in connection with this problem. Among others, the lattice approach [9], the use of effective potentials for composite operators [10], chiral perturbation theory [11], the QCD Sum Rule method (see for example [12]), and models like the Skyrmion approach and mixed constructions of hybrid models [13 16] can be mentioned.

In section II we obtain the fermionic mean number in terms of a trace involving the Green's function of the problem. This last has a nonregular behavior, which we study analytically in order to extract physical results.

In section III the difference between Gibbs and Helmholtz $(\mu=0$ case) free energy is obtained. Complementing this result with the Helmholtz energy previously evaluated in [2], we discuss its application to a simple quark-gluon droplet model [5, 17].

\section{THE GIBBS FREE ENERGY AND MEAN FERMIONIC NUMBER}

In this section we will be interested in the study of the Gibbs free energy of a massless fermionic field confined inside a static sphere of radius $R$ and obeying local boundary conditions corresponding to the M. I. T. bag model. 
This amounts to study the functional determinant of the Dirac operator under such boundary conditions, which is related to the Grand canonical partition function according to

$$
\Xi(T, R, \mu)=e^{-\beta G(T, R, \mu)} \sim \operatorname{Det}\left(D(\beta, R)-i \mu \gamma^{0}\right)_{b c} .
$$

Here

$$
D(\beta, R)=\frac{i}{\beta} \gamma^{0} \partial_{t}+\frac{i}{R} \vec{\gamma} \cdot \vec{\nabla}, \text { for } 0 \leq t,|\vec{x}| \leq 1,
$$

and " $b c$ " means that the operator is defined on a space of functions satisfying the local boundary conditions

$$
B \psi(t, \vec{x})=\left(\mathbf{1}_{4}+i \not h\right) \psi(t, \vec{x})=0, \text { for }|\vec{x}|=1,
$$

at the spatial edge and antiperiodic boundary conditions in the Euclidean time direction,

$$
\psi(1, \vec{x})=-\psi(0, \vec{x})
$$

as corresponds to the fermionic character of the field.

In the above expression, $\mu$ stands for the chemical potential and $\beta$ denotes the inverse of the temperature. In our conventions, the Euclidean gamma matrices are

$$
\gamma_{0}=i \rho^{3} \otimes \mathbf{1}_{2}, \vec{\gamma}=i \rho^{2} \otimes \vec{\sigma}, \gamma_{5}=\rho^{1} \otimes \mathbf{1}_{2},
$$

where $\rho^{k}, \sigma^{k}, k=1,2,3$, denote the Pauli matrices.

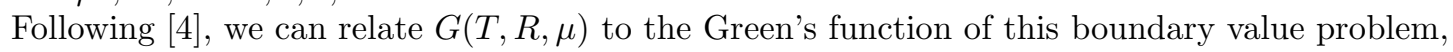

$$
\begin{gathered}
-\beta \frac{\partial G}{\partial \mu}(\beta, R, \mu)=\operatorname{Tr}\left\{\frac{\partial}{\partial \mu} \ln \left(D(\beta, R)-i \mu \gamma^{0}\right)_{b c}\right\} \\
=\operatorname{Tr}\left\{-i \gamma^{0} K_{b c}\left(t, x ; t^{\prime}, x^{\prime}\right)\right\},
\end{gathered}
$$

where $K_{b c}\left(t, x ; t^{\prime}, x^{\prime}\right)$ satisfies

$$
\begin{gathered}
\left(D(\beta, R)-i \mu \gamma^{0}\right) K_{b c}\left(t, \vec{x} ; t^{\prime}, \overrightarrow{x^{\prime}}\right)=\delta\left(\vec{x}-\overrightarrow{x^{\prime}}\right) \delta\left(t-t^{\prime}\right) \\
B K_{b c}\left(t, \vec{x} ; t^{\prime}, \overrightarrow{x^{\prime}}\right)=0, \text { for }|\vec{x}|=1, \\
K_{b c}\left(1, \vec{x} ; t^{\prime}, \overrightarrow{x^{\prime}}\right)=-K_{b c}\left(0, \vec{x} ; t^{\prime}, \overrightarrow{x^{\prime}}\right) .
\end{gathered}
$$

In fact, (6) gives us the particle mean number $N(\beta, \mu)=-\frac{\partial G}{\partial \mu}$.

As discussed in 㚪, the chemical potential $\mu$ can also be included in the boundary condition for the Euclidean time direction, through the invertible transformation

$$
\begin{gathered}
D(\beta, R)-i \mu \gamma^{0}=e^{\mu \beta t} D(\beta, R) e^{-\mu \beta t}, \\
K_{b c}\left(t, \vec{x} ; t^{\prime}, \overrightarrow{x^{\prime}}\right)=e^{\mu \beta t} k\left(t, \vec{x} ; t^{\prime}, \overrightarrow{x^{\prime}}\right) e^{-\mu \beta t \prime},
\end{gathered}
$$

where $k\left(t, \vec{x} ; t^{\prime}, \overrightarrow{x^{\prime}}\right)$ is the Green's function of the modified problem,

$$
\begin{gathered}
D(\beta, R) k\left(t, \vec{x} ; t^{\prime}, \overrightarrow{x^{\prime}}\right)=\delta\left(\vec{x}-\overrightarrow{x^{\prime}}\right) \delta\left(t-t^{\prime}\right) \\
B e^{\mu \beta t} k\left(t, \vec{x} ; t^{\prime}, \overrightarrow{x^{\prime}}\right)=0, \text { for }|\vec{x}|=1, \\
k\left(1, \vec{x} ; t^{\prime}, \overrightarrow{x^{\prime}}\right)+e^{-\mu \beta} k\left(0, \vec{x} ; t^{\prime}, \overrightarrow{x^{\prime}}\right)=0 .
\end{gathered}
$$

This function has the development

$$
k\left(t, x ; t^{\prime}, x^{\prime}\right)=R \sum_{n=-\infty}^{\infty} k_{n}\left(x, x^{\prime}\right) e^{-i \Omega_{n} \beta\left(t-t^{\prime}\right)}
$$


with the frequencies given by

$$
\begin{gathered}
\Omega_{n}=\omega_{n}-i \mu, \\
\omega_{n}=\frac{(2 n+1) \pi}{\beta}, \text { for } n \in \mathbf{Z},
\end{gathered}
$$

and $k_{n}\left(x ; x^{\prime}\right)$ satisfying

$$
\begin{gathered}
{\left[\Omega_{n} R i \rho^{3} \otimes \mathbf{1}_{2}-\rho^{2} \otimes \vec{\sigma} \cdot \vec{\nabla}\right] k_{n}\left(x ; x^{\prime}\right)=\delta^{(3)}\left(x-x^{\prime}\right) \rho^{0} \otimes \mathbf{1}_{2}} \\
B k_{n}\left(\vec{x} ; \overrightarrow{x^{\prime}}\right)=0
\end{gathered}
$$

It is convenient to define dimensionless parameters $\bar{\Omega}_{n}=R \Omega_{n}, \bar{\mu}=R \mu$ and $z=R / \beta$.

The solution of (9) can be expressed as the sum of a solution of the inhomogeneous equation, $k_{0}\left(t, x ; t^{\prime}, x^{\prime}\right)$, with a vanishing behavior at infinity, and a solution of the homogeneous equation, $\tilde{k}\left(t, x ; t^{\prime}, x^{\prime}\right)$, chosen in such a way that the complete Green's function $k\left(t, x ; t^{\prime}, x^{\prime}\right)$ obeys the boundary condition in (9). This splitting of the Green's function induces a similar separation for the mean fermionic number. In subsections II A and II B, we will considered both pieces separately.

\section{A. Fermionic mean number for the boundaryless case}

In this case the mean fermionic number is given by

$$
N_{0}=-\left.\frac{\partial G}{\partial \mu}\right|_{0}=\frac{1}{\beta} \operatorname{Tr}\left[-i \gamma^{0} e^{\mu \beta t} k_{0} e^{-\mu \beta t^{\prime}}\right]
$$

where $k_{0}$ corresponds to the Green's function of the modified problem (9), satisfying the temporal boundary condition and vanishing at spatial infinity,

$$
\begin{gathered}
k_{0}\left(t, x ; t^{\prime}, x^{\prime}\right)=R \sum_{n=-\infty}^{\infty} k_{0}^{(n)}\left(x, x^{\prime}\right) e^{-i \Omega_{n} \beta\left(t-t^{\prime}\right)} \\
k_{0}^{(n)}\left(\vec{x}, \overrightarrow{x^{\prime}}\right)=-\frac{1}{4 \pi} \frac{e^{-S_{n} \bar{\Omega}_{n}\left|\vec{x}-\overrightarrow{x^{\prime}}\right|}}{\left|\vec{x}-\overrightarrow{x^{\prime}}\right|}\left[\bar{\Omega}_{n} \gamma^{0}+\left(\frac{1}{\left|\vec{x}-\overrightarrow{x^{\prime}}\right|}-S_{n} \bar{\Omega}_{n}\right) \frac{i \vec{\gamma} \cdot\left(\vec{x}-\overrightarrow{x^{\prime}}\right)}{\left|\vec{x}-\overrightarrow{x^{\prime}}\right|}\right] .
\end{gathered}
$$

In the previous expression, $S_{n}=\operatorname{sign}(n+1 / 2)$.

In order to get $N_{0}$ we have to compute the trace in eq. (14). Due to the singular behavior of $k_{0}$ at the diagonal, we can take $t^{\prime}=t$, but we should keep $\overrightarrow{x^{\prime}} \neq \vec{x}$ up to the end of the calculation. In this way,

$$
\begin{aligned}
N_{0}= & \frac{1}{\beta} \int_{0}^{1} d t \int_{0}^{1} r^{2} d r \int_{\Omega} d \Omega\left\{\operatorname { t r } \left[-i \gamma^{0} R \sum_{n=-\infty}^{\infty}\left(-\frac{1}{4 \pi}\right) \frac{e^{-S_{n} \overline{\Omega_{n}}\left|\vec{x}-\overrightarrow{x^{\prime}}\right|}}{\left|\vec{x}-\overrightarrow{x^{\prime}}\right|} \times\right.\right. \\
& \left.\left.\left\{\bar{\Omega}_{n} \gamma^{0}+\left(\frac{1}{\left|\vec{x}-\overrightarrow{x^{\prime}}\right|}-S_{n} \bar{\Omega}_{n}\right) \frac{i \vec{\gamma} \cdot\left(\vec{x}-\overrightarrow{x^{\prime}}\right)}{\left|\vec{x}-\overrightarrow{x^{\prime}}\right|}\right\}\right]\right\}_{\overrightarrow{x^{\prime}} \rightarrow \vec{x}} .
\end{aligned}
$$

The second term in the integrand of (16) vanishes because of the properties of Dirac matrices. For the first one, with $\epsilon=\left|\vec{x}-\overrightarrow{x^{\prime}}\right|$, we get 


$$
\begin{array}{r}
N_{0}=\lim _{\epsilon \rightarrow 0^{+}}-\frac{4}{3} \frac{i R}{\epsilon} \sum_{n=-\infty}^{\infty} \bar{\Omega}_{n} e^{-\epsilon S_{n} \bar{\Omega}_{n}}= \\
-\left.\frac{8}{3 \beta} \frac{R}{\epsilon} \frac{\partial}{\partial \epsilon} \Im \sum_{n=0}^{\infty} e^{-\epsilon \bar{\Omega}_{n}}\right|_{\epsilon \rightarrow 0^{+}}
\end{array}
$$

So,

$$
N_{0}=\frac{4 \pi}{9}\left(\bar{\mu} z^{2}+\frac{\bar{\mu}^{3}}{\pi^{2}}\right)
$$

in agreement with the value for a free fermionic gas, as can be seen, for instance, in Ref. [18.

\section{B. Influence of the boundary conditions on the fermionic mean number}

For the solution of the homogeneous equation required to adjust the boundary conditions for the full Green's function, we propose the development

$$
\tilde{k}^{(n)}\left(\vec{x}, \overrightarrow{x^{\prime}}\right)=\sum_{k=0}^{3} \rho^{k} \otimes a^{k}\left(\vec{x}, \overrightarrow{x^{\prime}}\right)
$$

and write $k_{0}^{(n)}\left(\vec{x}, \overrightarrow{x^{\prime}}\right)$ as

$$
k_{0}^{(n)}\left(\vec{x}, \overrightarrow{x^{\prime}}\right)=\sum_{k=0}^{3} \rho^{k} \otimes A^{k}\left(\vec{x}, \overrightarrow{x^{\prime}}\right)
$$

Concerning the $A^{k}$ coefficients, $A^{0}$ and $A^{1}$ vanish, since they are the solutions of an everywhere regular homogeneous problem. For the other coefficients we have

$$
\begin{gathered}
A^{2}\left(\vec{x}, \overrightarrow{x^{\prime}}\right)=-\frac{\vec{\sigma} \cdot \vec{\nabla}}{i \bar{\Omega}_{n}} A^{3}\left(\vec{x}, \overrightarrow{x^{\prime}}\right) \\
{\left[\nabla^{2}+\left(i \bar{\Omega}_{n}\right)^{2}\right] A^{3}\left(\vec{x}, \overrightarrow{x^{\prime}}\right)=i \bar{\Omega}_{n} \delta^{(3)}\left(\vec{x}, \overrightarrow{x^{\prime}}\right) \mathbf{1}_{4} .}
\end{gathered}
$$

Following [19], we get $A^{3}$ as an expansion in terms of spinorial spherical harmonics,

$$
A^{3}\left(\vec{x}, \overrightarrow{x^{\prime}}\right)=\sum_{j, l, l^{\prime}, m} A_{j, l, l^{\prime}, m}^{3}\left(r, r^{\prime}\right) \phi_{j, l, m}(\Omega) \phi_{j, l^{\prime}, m}^{\dagger}\left(\Omega^{\prime}\right),
$$

where

$$
A_{j, l, l^{\prime}, m}^{3}\left(r, r^{\prime}\right)=\delta_{l, l^{\prime}} i S_{n} \bar{\Omega}_{n}^{2} j_{l}\left(i S_{n} \bar{\Omega}_{n} r_{<}\right) h_{l^{\prime}}^{(1)}\left(i S_{n} \bar{\Omega}_{n} r_{>}\right) .
$$

In eq. (24), $j_{l}(x)$ and $h_{l}^{(1)}(x)$ are the spherical Bessel and Hankel functions, respectively, and $r_{<}=\operatorname{Min}\left(r, r^{\prime}\right)$ $\left(r_{>}=\operatorname{Max}\left(r, r^{\prime}\right)\right)$.

For the coefficients $a^{k}$ in eq. (19), we get

$$
\begin{gathered}
a^{2}=-\frac{\vec{\sigma} \cdot \vec{\nabla}}{i \bar{\Omega}_{n}} a^{3}, \quad a^{1}=-\frac{\vec{\sigma} \cdot \vec{\nabla}}{i \bar{\Omega}_{n}} a^{0}, \\
{\left[\nabla^{2}+\left(i \bar{\Omega}_{n}\right)^{2}\right] a^{3}=0, \quad\left[\nabla^{2}+\left(i \bar{\Omega}_{n}\right)^{2}\right] a^{0}=0,}
\end{gathered}
$$

while the boundary conditions at $r=R$ imply 


$$
\begin{aligned}
\vec{\sigma} \cdot \hat{r} a^{0}-a^{2} & =A^{2}, \\
\vec{\sigma} \cdot \hat{r} a^{1}-i a^{3} & =i A^{3} .
\end{aligned}
$$

Using the following Ansatz,

$$
a^{k}\left(\vec{x}, \overrightarrow{x^{\prime}}\right)=\sum_{j, l, l^{\prime}, m} C_{j, l, l^{\prime}, m}^{k} j_{l}\left(i S_{n} \bar{\Omega}_{n} r\right) j_{l}^{\prime}\left(i S_{n} \bar{\Omega}_{n} r^{\prime}\right) \phi_{j, l, m}(\Omega) \phi_{j, l^{\prime}, m}^{\dagger}\left(\Omega^{\prime}\right),
$$

we finally get

$$
\begin{gathered}
k_{n}\left(\vec{x}, \vec{x}^{\prime}\right)=\left(i \bar{\Omega}_{n}\right)^{2} \sum_{j, l, l^{\prime}, m} \\
{\left[\left(\rho^{0} \delta_{l, l^{\prime}} i^{\bar{l}-l} d_{j}+\rho^{1} \delta_{\bar{l}, l^{\prime}} S_{n} i^{\bar{l}-l} d_{j}-\rho^{2} \delta_{\bar{l}, l^{\prime}} c_{j}+\rho^{3} i S_{n} \delta_{l, l^{\prime}} c_{j}\right) j_{l}\left(i S_{n} \bar{\Omega}_{n} r\right) j_{l^{\prime}}\left(i S_{n} \bar{\Omega}_{n} r^{\prime}\right)\right.} \\
\left.+\left(\rho^{2} \delta_{\bar{l}, l^{\prime}}-\rho^{3} i S_{n} \delta_{l, l^{\prime}}\right) j_{l}\left(i S_{n} \bar{\Omega}_{n} r_{<}\right) h_{l^{\prime}}^{(1)}\left(i S_{n} \bar{\Omega}_{n} r_{>}\right)\right] \otimes \phi_{j, l, m}(\Omega) \phi_{j, l^{\prime}, m}^{\dagger}\left(\Omega^{\prime}\right),
\end{gathered}
$$

where $c_{j}$ and $d_{j}$ are given by

$$
\begin{gathered}
c_{j}=\frac{j_{j+1 / 2}\left(i S_{n} \bar{\Omega}_{n}\right) h_{j+1 / 2}^{(1)}\left(i S_{n} \bar{\Omega}_{n}\right)-j_{j-1 / 2}\left(i S_{n} \bar{\Omega}_{n}\right) h_{j-1 / 2}^{(1)}\left(i S_{n} \bar{\Omega}_{n}\right)}{j_{j+1 / 2}^{2}\left(i S_{n} \bar{\Omega}_{n}\right)-j_{j-1 / 2}^{2}\left(i S_{n} \bar{\Omega}_{n}\right)}, \\
d_{j}=\frac{-i / \bar{\Omega}_{n}^{2}}{j_{j+1 / 2}^{2}\left(i S_{n} \bar{\Omega}_{n}\right)-j_{j-1 / 2}^{2}\left(i S_{n} \bar{\Omega}_{n}\right)} .
\end{gathered}
$$

The correction to the fermionic mean number due to the boundary conditions is

$$
\tilde{N}=\frac{1}{\beta} \operatorname{Tr}\left[-i \gamma^{0} e^{\mu \beta t} \tilde{k} e^{-\mu \beta t^{\prime}}\right] .
$$

Once again, in order to get a well defined result, we must proceed carefully when treating the kernel of this operator at the diagonal. We can take $t^{\prime}=t$ and $\Omega^{\prime}=\Omega$ safely, while keeping $r^{\prime}=r(1-\epsilon)$, with $\epsilon>0$. We thus get

$$
\begin{gathered}
\tilde{N}=2 z \lim _{\epsilon \rightarrow 0^{+}} \sum_{n=-\infty}^{\infty} i S_{n}\left(i \bar{\Omega}_{n}\right)^{2} \sum_{j, m} c_{j} \int_{0}^{1} r^{2} d r \times \\
{\left[j_{j+1 / 2}^{2}\left(i S_{n} \bar{\Omega}_{n} r\right) j_{j+1 / 2}^{2}\left(i S_{n} \bar{\Omega}_{n} r^{\prime}\right)+j_{j-1 / 2}^{2}\left(i S_{n} \bar{\Omega}_{n} r\right) j_{j-1 / 2}^{2}\left(i S_{n} \bar{\Omega}_{n} r^{\prime}\right)\right]_{r^{\prime}=r(1-\epsilon)} .}
\end{gathered}
$$

After a straightforward calculation of the radial integral, we obtain the following expresion

$$
\begin{gathered}
\tilde{N}=\lim _{\epsilon \rightarrow 0^{+}} \sum_{n=-\infty}^{\infty} N_{n, k}(z, \bar{\mu} ; \epsilon) \\
=-2 i z \lim _{\epsilon \rightarrow 0^{+}} \sum_{n=-\infty}^{\infty} \sum_{k=0}^{\infty} \frac{(1-\epsilon)^{-3 / 2}}{\epsilon} \frac{1}{\bar{\Omega}_{n}}(2 \nu+1) \frac{d_{\nu, n}^{\epsilon}-d_{\nu, n}+\epsilon\left(d_{\nu, n}-\nu\right)}{\left(d_{\nu, n}-\nu\right)^{2}+\bar{\Omega}_{n}^{2}} \times \\
\frac{I_{\nu}\left(S_{n} \bar{\Omega}_{n}(1-\epsilon)\right)}{I_{\nu}\left(S_{n} \bar{\Omega}_{n}\right)}\left[\bar{\Omega}_{n}^{2} I_{\nu}^{\prime}\left(S_{n} \bar{\Omega}_{n}\right) K_{\nu}^{\prime}\left(S_{n} \bar{\Omega}_{n}\right)-S_{n} \bar{\Omega}_{n} \nu\left(I_{\nu}\left(S_{n} \bar{\Omega}_{n}\right) K_{\nu}\left(S_{n} \bar{\Omega}_{n}\right)\right)^{\prime}\right. \\
\left.+\left(\nu^{2}+\bar{\Omega}_{n}^{2}\right) I_{\nu}\left(S_{n} \bar{\Omega}_{n}\right) K_{\nu}\left(S_{n} \bar{\Omega}_{n}\right)\right]
\end{gathered}
$$


where

$$
d_{\nu, n}^{\epsilon}=\left.S_{n} \bar{\Omega}_{n}(1-\epsilon) \frac{d}{d x} \log I_{\nu}(x)\right|_{x=S_{n} \bar{\Omega}_{n}(1-\epsilon)}, \quad \nu=k+\frac{1}{2}
$$

and $d_{\nu, n}=\left.d_{\nu, n}^{\epsilon}\right|_{\epsilon=0}$.

The double series (32) is not absolutely convergent for $\epsilon=0$, so we must keep $\epsilon>0$ in the general term. A way to isolate such singular behavior consists in the substraction of the first terms in the asymptotic (Debye) expansion of $N_{k, n}, \Delta_{k, n}^{M}$ (see, for example, [2]). The difference

$$
\left|N_{k, n}(z, \bar{\mu} ; \epsilon)-\Delta_{k, m}^{M}(z, \bar{\mu} ; \epsilon)\right| \asymp \mathcal{O}\left(\frac{1}{\rho^{M+1}}\right),
$$

where $\rho^{2}=\bar{\Omega}_{n}^{2}+\nu^{2}$ can be considered as a "radial" variable in the double series.

So, we can write

$$
\begin{gathered}
\tilde{N}=\tilde{N}_{1}+\tilde{N}_{2}= \\
\sum_{n=-\infty}^{\infty} \sum_{k=0}^{\infty}\left(N_{k, n}(z, \bar{\mu} ; \epsilon)-\Delta_{k, m}^{M}(z, \bar{\mu} ; \epsilon)\right)_{\epsilon=0}+\lim _{\epsilon \rightarrow 0} \sum_{n=-\infty}^{\infty} \sum_{k=0}^{\infty} \Delta_{k, n}^{M}(z, \bar{\mu} ; \epsilon) .
\end{gathered}
$$

In the first term of (35), for $M \geq 3$, the limit $\epsilon \rightarrow 0$ can be taken for the general term, inside the (absolutely and uniformly convergent) double sum. The nonregular behavior of the series is isolated in the second term of (35).

Our calculation strategy consists in the numerical evaluation of the first term of (35), and the analytical study of the second one. Notice that we may take $M>3$ in order to improve the numerical convergence of the first term.

\section{Asymptotic (Debye) expansions}

Introducing the Debye expansion for the modified Bessel functions [20], we can write

$$
\Delta_{k, m}^{M}=\bar{\Omega}_{n} D(\nu, t, \epsilon) E(\nu, t, \epsilon),
$$

with

$$
\begin{aligned}
& D(\nu, t, \epsilon)=\left\{(-2 i z)(1-\epsilon)^{-3 / 2} \quad \frac{2 \nu+1}{2 \nu} \quad \frac{d_{\nu, n}^{\epsilon}-d_{\nu, n}+\epsilon\left(d_{\nu, n}-\nu\right)}{\epsilon} \times\right. \\
& \frac{1}{d_{\nu, n}^{2}-2 \nu d_{\nu, n}+\nu^{2} / t^{2}} \quad f^{-1 / 4}(\epsilon, t) \quad \frac{\left.S_{u}^{+}\left(\nu, t f^{(}-1 / 2\right)(\epsilon, t)\right)}{S_{u}^{+}(\nu, t)} \times \\
& \left.\frac{t}{1-t^{2}}\left[S_{u}^{+}(\nu, t) S_{u}^{-}(\nu, t)-S_{v}^{+}(\nu, t) S_{v}^{-}(\nu, t)+t\left(S_{u}^{+}(\nu, t) S_{v}^{-}(\nu, t)-S_{u}^{-}(\nu, t) S_{v}^{+}(\nu, t)\right)\right]\right\}\left.\right|_{M}
\end{aligned}
$$

where $\left.\right|_{M}$ stands for a consistent expansion up to the order $\rho^{-M}$, and

$$
E(\nu, t, \epsilon)=\exp \left(\frac{\nu}{t}\left(f^{1 / 2}(\epsilon, t)-1\right)+\nu \log \left((1-\epsilon) \frac{t+1}{t+f^{1 / 2}(\epsilon, t)}\right)\right) .
$$

In the expressions above,

$$
t=\frac{\nu}{\rho}, \quad f(\epsilon, t)=1-\epsilon(2-\epsilon)\left(1-t^{2}\right)
$$




$$
\begin{aligned}
& S_{u}^{+}(\nu, t)=1+\sum_{k=1}^{\infty} \frac{u_{k}(t)}{\nu^{k}}, \\
& S_{u}^{-}(\nu, t)=1+\sum_{k=1}^{\infty}(-1)^{k} \frac{u_{k}(t)}{\nu^{k}}, \\
& S_{v}^{+}(\nu, t)=1+\sum_{k=1}^{\infty} \frac{v_{k}(t)}{\nu^{k}}, \\
& S_{v}^{-}(\nu, t)=1+\sum_{k=1}^{\infty}(-1)^{k} \frac{v_{k}(t)}{\nu^{k}},
\end{aligned}
$$

with $u_{k}(t)$ and $v_{k}(t)$ being the polynomials appearing in the Debye expansion of Bessel functions, as defined as in Ref. [20].

It is easy to see that

$$
E(\nu, t, \epsilon)=\exp \left(-\epsilon \frac{\nu}{t}\right)\left(1+O\left(\epsilon^{2}\right)\right)
$$

So, the corrections to the fermionic mean number due to the boundary conditions (35) can be written as

$$
\begin{gathered}
\tilde{N}_{1}=\sum_{n=-\infty}^{\infty} \sum_{k=0}^{\infty}\left[N_{n, k}(z, \bar{\mu} ; \epsilon=0)-\bar{\Omega}_{n} D(\nu, t, \epsilon=0)\right], \\
\tilde{N}_{2}=\left[\sum_{n=-\infty}^{\infty} \sum_{k=0}^{\infty} \bar{\Omega}_{n} D(\nu, t, \epsilon) E(\nu, t, \epsilon)\right]_{\epsilon \rightarrow 0^{+}} .
\end{gathered}
$$

The first term, $\tilde{N}_{1}$, will be numerically evaluated from the expression

$$
\begin{gathered}
N_{n, k}(z, \bar{\mu}, \epsilon=0)= \\
(-2 i z) \bar{\Omega}_{n}(2 \nu+1) \frac{d_{\nu, n}^{2}-\nu^{2} / t^{2}+d_{\nu, n}-\nu}{d_{\nu, n}^{2}-2 \nu d_{\nu, n}+\nu^{2} / t^{2}}\left[I_{\nu}^{\prime}\left(S_{n} \bar{\Omega}_{n}\right) K_{\nu}^{\prime}\left(S_{n} \bar{\Omega}_{n}\right)\right. \\
\left.-\frac{\nu}{S_{n} \bar{\Omega}_{n}} \nu\left(I_{\nu}\left(S_{n} \bar{\Omega}_{n}\right) K_{\nu}\left(S_{n} \bar{\Omega}_{n}\right)\right)^{\prime}+\frac{\nu^{2}+\bar{\Omega}_{n}^{2}}{\bar{\Omega}_{n}^{2}} I_{\nu}\left(S_{n} \bar{\Omega}_{n}\right) K_{\nu}\left(S_{n} \bar{\Omega}_{n}\right)\right]
\end{gathered}
$$

and from the asymptotic expansion $D$, at $\epsilon=0$. For computational convenience we take $M=6$; the corresponding expression for $D$ is

$$
\begin{gathered}
D(\nu, t, \epsilon=0)=-2 i z\left[\frac{-t^{5}}{2 \nu^{2}}+\frac{1}{\nu^{3}}\left(\frac{-3 t^{5}}{4}-\frac{t^{6}}{2}+\frac{5 t^{7}}{4}+\frac{3 t^{8}}{4}\right)\right. \\
+\frac{1}{\nu^{4}}\left(\frac{-3 t^{5}}{8}-\frac{3 t^{6}}{4}+\frac{11 t^{7}}{16}+\frac{27 t^{8}}{8}+\frac{7 t^{9}}{2}-3 t^{10}-\frac{71 t^{11}}{16}\right) \\
+\frac{1}{\nu^{5}}\left(\frac{-t^{5}}{16}-\frac{3 t^{6}}{8}-\frac{27 t^{7}}{32}+\frac{9 t^{8}}{4}+\frac{483 t^{9}}{32}+\frac{25 t^{10}}{8}-\frac{1211 t^{11}}{32}-\frac{285 t^{12}}{16}+\frac{781 t^{13}}{32}+\frac{213 t^{14}}{16}\right) \\
+\frac{1}{\nu^{6}}\left(\frac{-t^{6}}{16}-\frac{47 t^{7}}{64}-\frac{3 t^{8}}{4}+\frac{2909 t^{9}}{256}+\frac{473 t^{10}}{16}-\frac{531 t^{11}}{64}\right.
\end{gathered}
$$




$$
\left.\left.-\frac{4255 t^{12}}{32}-\frac{14795 t^{13}}{128}+\frac{6297 t^{14}}{32}+\frac{481 t^{15}}{2}-\frac{1491 t^{16}}{16}-\frac{32799 t^{17}}{256}\right)\right]
$$

The second term, $\tilde{N}_{2}$, will be studied analytically in the following. Taking into acount (43), we have

$$
\tilde{N}_{2}=\left[\sum_{n=-\infty}^{\infty} \sum_{k=0}^{\infty} \bar{\Omega}_{n} D(\nu, t, \epsilon=0) e^{-\epsilon \nu / t}(1+O(\epsilon))\right]_{\epsilon \rightarrow 0^{+}},
$$

Now, employing (47), Equation (48) can be naturally expressed in terms of the double series

$$
s(p, q ; \epsilon)=\sum_{n=-\infty}^{\infty} \sum_{k=0}^{\infty}(-2 z) i \bar{\Omega}_{n} e^{-\epsilon \rho} \rho^{-p} \nu^{q},
$$

(where, for convenience, we omit the dependence on $\bar{\mu}$ and $z$ ) obtaining

$$
\begin{aligned}
\tilde{N}_{2}= & \lim _{\epsilon \rightarrow 0^{+}}\left[\left\{\frac{-s(5,0 ; \epsilon)}{16}-\frac{3 s(5,1 ; \epsilon)}{8}-\frac{3 s(5,2 ; \epsilon)}{4}-\frac{s(5,3 ; \epsilon)}{2}\right.\right. \\
& \quad \frac{s(6,0 ; \epsilon)}{16}-\frac{3 s(6,1 ; \epsilon)}{8}-\frac{3 s(6,2 ; \epsilon)}{4}-\frac{s(6,3 ; \epsilon)}{2} \\
& -\frac{47 s(7,1 ; \epsilon)}{64}-\frac{27 s(7,2 ; \epsilon)}{32}+\frac{11 s(7,3 ; \epsilon)}{16}+\frac{5 s(7,4 ; \epsilon)}{4} \\
& -\frac{3 s(8,2 ; \epsilon)}{4}+\frac{9 s(8,3 ; \epsilon)}{4}+\frac{27 s(8,4 ; \epsilon)}{8}+\frac{3 s(8,5 ; \epsilon)}{4} \\
+ & \frac{2909 s(9,3 ; \epsilon)}{256}+\frac{483 s(9,4 ; \epsilon)}{32}+\frac{7 s(9,5 ; \epsilon)}{2}+\frac{473 s(10,4 ; \epsilon)}{16} \\
+ & \frac{25 s(10,5 ; \epsilon)}{8}-3 s(10,6 ; \epsilon)-\frac{531 s(11,5 ; \epsilon)}{64}-\frac{1211 s(11,6 ; \epsilon)}{32} \\
- & \frac{71 s(11,7 ; \epsilon)}{16}-\frac{4255 s(12,6 ; \epsilon)}{32}-\frac{285 s(12,7 ; \epsilon)}{16}-\frac{14795 s(13,7 ; \epsilon)}{128} \\
+ & \frac{781 s(13,8 ; \epsilon)}{32}+\frac{6297 s(14,8 ; \epsilon)}{32}+\frac{213 s(14,9 ; \epsilon)}{16}+\frac{481 s(15,9 ; \epsilon)}{2} \\
+ & \left.\left.\frac{32799 s(17,11 ; \epsilon)}{256}\right\}(1+\mathcal{O}(\epsilon))\right]
\end{aligned}
$$

In the following section we will prove that $s(p, q ; \epsilon)$ has a finite $\epsilon \rightarrow 0^{+}$-limit, which allows for the evaluation of (50). 
In this section we study the double sums defined in equation (49), which can be written as

$$
s(p, q ; \epsilon)=\sum_{k=0}^{\infty} \nu^{q} \mathcal{S}_{p}(\nu, \epsilon),
$$

where

$$
\mathcal{S}_{p}(\nu, \epsilon)=(-2 z) \sum_{n=-\infty}^{\infty} \frac{i \bar{\Omega}_{n} e^{-\epsilon \sqrt{\nu^{2}+\bar{\Omega}_{n}^{2}}}}{\left(\nu^{2}+\bar{\Omega}_{n}^{2}\right)^{p / 2}} .
$$

It is easily seen that the $\mathcal{S}_{p}(\nu, \epsilon)$ are odd-functions of $\bar{\mu}$, and satisfy the recursion relations

$$
\begin{gathered}
\mathcal{S}_{p-1}(\nu, \epsilon)=-\frac{\partial}{\partial \epsilon} \mathcal{S}_{p}(\nu, \epsilon), \\
\mathcal{S}_{p}(\nu, \epsilon)=-\frac{1}{m-2}\left[\epsilon \mathcal{S}_{p-1}(\nu, \epsilon)+\frac{1}{\nu} \frac{\partial \mathcal{S}_{p-2}(\nu, \epsilon)}{\partial \nu}\right], \quad \text { for } \quad m \neq 2,
\end{gathered}
$$

which allow the computation of any $\mathcal{S}_{p}(\nu, \epsilon)$ from the knowledge of $\mathcal{S}_{2}(\nu, \epsilon)$. The last series will be determined in what follows.

Making use of the Poisson formula [20], $\mathcal{S}_{2}(\nu, \epsilon)$ can be transformed into the series

$$
\mathcal{S}_{2}(\nu, \epsilon)=\sum_{\ell=-\infty}^{\infty} C_{\ell}
$$

where the coefficients are given by

$$
C_{\ell}=\frac{(-1)^{\ell}}{\pi} 2 \Im \int_{0}^{\infty} d x \frac{x-i \bar{\mu}}{(x-i \bar{\mu})^{2}+\nu^{2}} e^{i \ell x / z} e^{-\epsilon \sqrt{(x-i \bar{\mu})^{2}+\nu^{2}}} .
$$

It is convenient to treat the terms with $\ell=0, \ell>0$ and $\ell<0$ separately. The corresponding integrals can be exactly computed in each case, by adequately extending the integration path on the complex $x$-plane. For $\bar{\mu}>0$, one gets

$$
\begin{gathered}
C_{0}=H(\bar{\mu}-\nu)\left[1-\frac{2}{\pi} \int_{\nu}^{\bar{\mu}} d x \frac{x}{x^{2}-\nu^{2}} \sin \left(\epsilon \sqrt{x^{2}-\nu^{2}}\right)\right], \\
C_{\ell>0}=(-1)^{\ell}\left\{e^{-\ell(\bar{\mu}+\nu) / z}-\frac{2}{\pi} \int_{\nu}^{\infty} d x \frac{x}{x^{2}-\nu^{2}} e^{-\ell(\bar{\mu}+x) / z} \sin \left(\epsilon \sqrt{x^{2}-\nu^{2}}\right)+\right. \\
\left.H(\bar{\mu}-\nu)\left[e^{-\ell(\bar{\mu}-\nu) / z}-\frac{2}{\pi} \int_{\nu}^{\bar{\mu}} d x \frac{x}{x^{2}-\nu^{2}} e^{-\ell(\bar{\mu}-x) / z} \sin \left(\epsilon \sqrt{x^{2}-\nu^{2}}\right)\right]\right\}, \\
C_{\ell<0}=(-1)^{\ell}\left\{\frac{2}{\pi} H(\bar{\mu}-\nu) \int_{\bar{\mu}}^{\infty} d x \frac{x}{x^{2}-\nu^{2}} e^{-\ell(\bar{\mu}-x) / z} \sin \left(\epsilon \sqrt{x^{2}-\nu^{2}}\right)+\right. \\
\left.H(\nu-\bar{\mu})\left[-e^{-\ell(\bar{\mu}-\nu) / z}+\frac{2}{\pi} \int_{\nu}^{\infty} d x \frac{x}{x^{2}-\nu^{2}} e^{-\ell(\bar{\mu}-x) / z} \sin \left(\epsilon \sqrt{x^{2}-\nu^{2}}\right)\right]\right\},
\end{gathered}
$$

where $H(x)$ stands for the Heaviside step function. 
Finally, the sum over the index $\ell$ can be perfomed to get

$$
\begin{gathered}
\mathcal{S}_{2}(\nu, \epsilon)=\frac{1}{1+e^{-(\bar{\mu}-\nu) / z}}-\frac{1}{1+e^{(\bar{\mu}+\nu) / z}}- \\
\frac{2}{\pi} \int_{0}^{\infty} \frac{d u}{u}\left[\frac{1}{1+e^{-\bar{\mu} / z} e^{\frac{\nu}{z} \sqrt{u^{2}+1}}}-\frac{1}{1+e^{+\bar{\mu} / z} e^{\frac{\nu}{z} \sqrt{u^{2}+1}}}\right] \sin (\epsilon \nu u) .
\end{gathered}
$$

Now, the expressions for $\mathcal{S}_{p \neq 2}(\nu, \epsilon)$ can be inmediately obtained employing (53) and (54).

All $\mathcal{S}_{p}(\nu, \epsilon)$ so defined are regular at $\epsilon=0$, and exponentially vanishing with $\nu$ (even at $\epsilon=0$ ). Such behavior guaranties the uniform convergence of the $k$-series in expression (51), allowing to take the $\epsilon \rightarrow 0$ limit of $\mathcal{S}_{p}(\nu, \epsilon)$ therein,

$$
s(p, q ; \epsilon=0)=\sum_{k=0}^{\infty} \nu^{q} \mathcal{S}_{p}(\nu, \epsilon=0) .
$$

This limit also simplifies the recursion relations leading to

$$
\begin{aligned}
& \mathcal{S}_{2 \kappa}(\nu, 0)=\frac{1}{[2(\kappa-1)] ! !}\left(-\frac{1}{\nu} \frac{\partial}{\partial \nu}\right)^{\kappa-1} \mathcal{S}_{2}(\nu, 0) \\
& \mathcal{S}_{2 \kappa+1}(\nu, 0)=\frac{1}{[2 \kappa-1] ! !}\left(-\frac{1}{\nu} \frac{\partial}{\partial \nu}\right)^{\kappa} \mathcal{S}_{1}(\nu, 0),
\end{aligned}
$$

where

$$
\begin{gathered}
\mathcal{S}_{1}(\nu, 0)=\frac{2 \nu}{\pi} \int_{0}^{\infty} d u\left[\frac{1}{1+e^{-\bar{\mu} / z} e^{\frac{\nu}{z} \sqrt{u^{2}+1}}}-\frac{1}{1+e^{+\bar{\mu} / z} e^{\frac{\nu}{z} \sqrt{u^{2}+1}}}\right] \\
\mathcal{S}_{2}(\nu, 0)=\frac{1}{1+e^{-(\bar{\mu}-\nu) / z}}-\frac{1}{1+e^{(\bar{\mu}+\nu) / z}} .
\end{gathered}
$$

Equations (62) to (65) lead to exact expressions for $\mathcal{S}_{p}(\nu, 0)$, allowing for the numerical evaluation of $s(p, q ; \epsilon=0)$. We can also obtain analytically an expansion of $s(p, q ; \epsilon=0)$ for large values of $z=R T$. In fact, for $p \geq 4$ it is straightforward to get

$$
s(p, q ; \epsilon=0)=(-2 z) \frac{1}{p-2} \frac{\partial}{\partial \bar{\mu}} 2 \Re \sigma_{p-2, q},
$$

where

$$
\sigma_{p, q}=\sum_{k=0}^{\infty} \nu^{q} \sum_{n=0}^{\infty} \rho^{-p}
$$

Making use of the integral representation

$$
\rho^{-p}=\frac{1}{\Gamma(p / 2)} \int_{0}^{\infty} d \tau \tau^{\frac{p}{2}-1} e^{-\tau \rho^{2}}
$$

we obtain

$$
\sigma_{p, q}=\frac{1}{\Gamma(p / 2)} \int_{0}^{\infty} d \tau \tau^{\frac{1}{2}(p-q)-1}\left(\sum_{k=0}^{\infty}\left(\tau \nu^{2}\right)^{q / 2} e^{-\tau \nu^{2}}\right)\left(\sum_{n=0}^{\infty} e^{-\tau(2 \pi z)^{2}\left[n+\left(\frac{1}{2}-i \frac{\bar{\mu}}{2 \pi z}\right)\right]^{2}}\right) .
$$

In this expression, for large $z$ and for each $n$, high values of $\tau$ are strongly suppressed by the exponential factor $e^{-\tau(2 \pi z)\left(n+\frac{1}{2}\right)}$. So, we can use the Euler-Mac Laurin expansion [20] to evaluate the factor 


$$
\sum_{k=0}^{\infty}\left(\tau \nu^{2}\right)^{q / 2} e^{-\tau \nu^{2}}=\frac{1}{2} \frac{1}{\sqrt{\tau}} \Gamma\left(\frac{d+1}{2}\right)+\left.\frac{1}{2} \sum_{\ell=1}^{\infty} \frac{(-1)^{\ell+1}}{(\ell+1) !}\left[\frac{1}{2^{\ell}}-2 B_{\ell+1}\right] \tau^{\ell / 2} \frac{d^{\ell}}{d x^{\ell}}\left(x^{q} e^{-x^{2}}\right)\right|_{x=\frac{\sqrt{\tau}}{2}} .
$$

Replacing in (58), solving the $\tau$-integral and the remaining $n$-sum, we get

$$
\begin{gathered}
\left.s(p, q ; \epsilon=0)\right|_{z \gg 1}= \\
\frac{(-2 z)}{p-2} \frac{\partial}{\partial \bar{\mu}} \Re\left[\left(\frac{1}{2 \pi z}\right)^{p-q-3} \frac{\Gamma\left(\frac{p-q-3}{2}\right) \Gamma\left(\frac{q+1}{2}\right)}{\Gamma\left(\frac{p-2}{2}\right)} \zeta\left(p-q-3, \frac{1}{2}-i \frac{\bar{\mu}}{2 \pi z}\right)+\right. \\
\left.2 \sum_{\ell=0}^{\infty} \frac{(-1)^{\ell}}{\ell !}\left(\frac{1}{2 \pi z}\right)^{p-2+2 \ell} \frac{\Gamma\left(\frac{p-2+2 \ell}{2}\right)}{\Gamma\left(\frac{p-2}{2}\right)} \zeta\left(p-2+2 \ell, \frac{1}{2}-i \frac{\bar{\mu}}{2 \pi z}\right) \zeta\left(-(q+2 \ell), \frac{1}{2}\right)\right],
\end{gathered}
$$

for $p \geq 4$.

\section{NUMERICAL EVALUATIONS AND APPLICATIONS}

The results obtained in the previous sections allow for the complete evaluation of the mean fermionic number and the $\mu$-dependent part of the Gibbs free energy for the fermionic field we are considering.

In order to obtain $N$, at fixed $z$, we can numerically evaluate $\tilde{N}_{1}$ from equations (44), (46) and (47), and $\tilde{N}_{2}$ from (50) and (61) to (65).

Adding these contributions to $N_{0}$, equation (18), we obtain the fermionic mean number. The result is shown in Fig. 1, for $z=1 / 8,1 / 4$

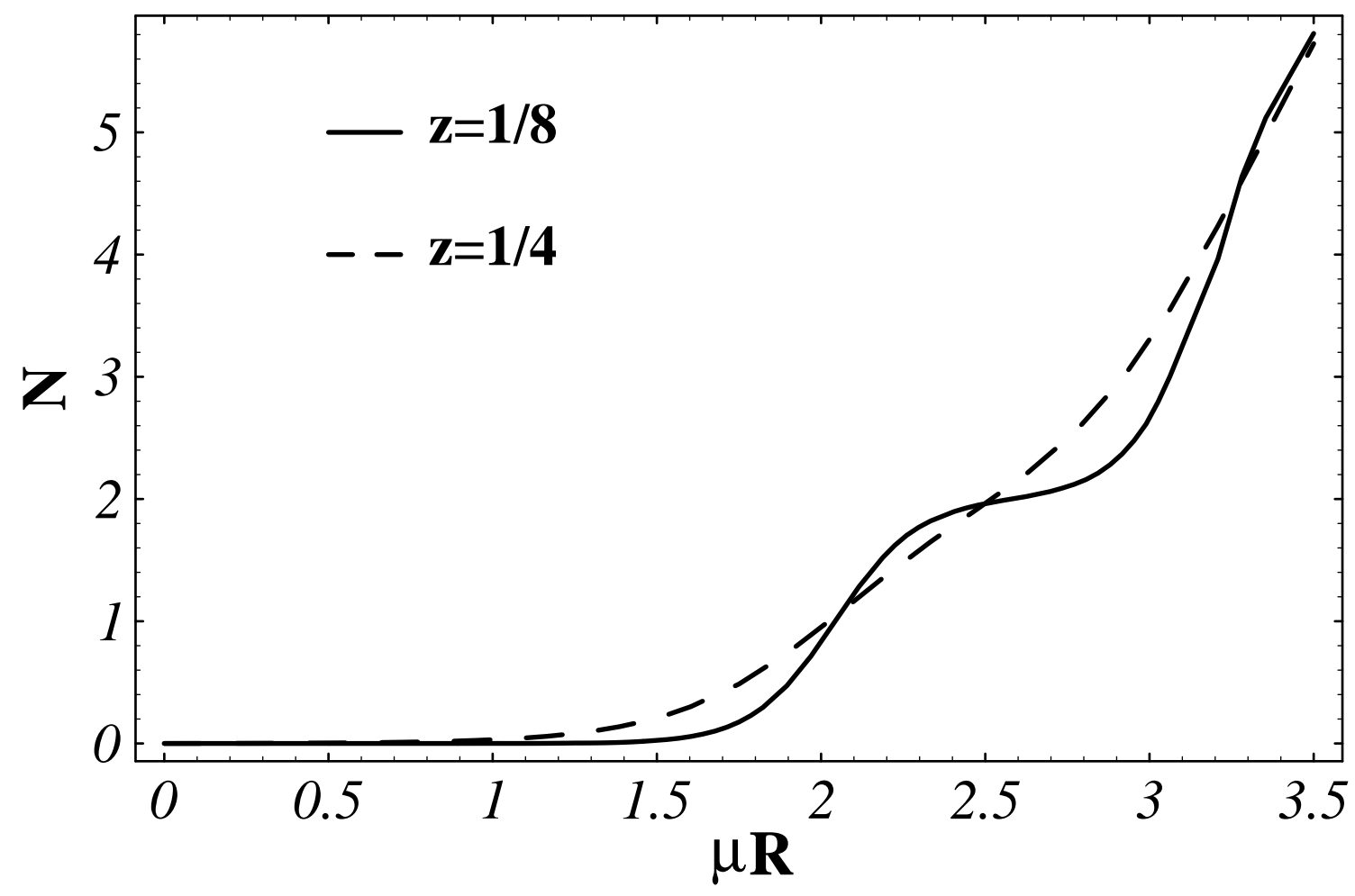

FIG. 1. Fermionic mean number for $z=1 / 8,1 / 4$ 
Notice that $N(z, \bar{\mu})$ has the expected behavior with $\bar{\mu}$, since it shows steps at the adimensionalized eigenvalues of the Dirac Hamiltonian 21$]$.

Numerical evaluations become more difficult when $z$ grows up. But, in the $z>1$ region, the asymptotic approximation for $\tilde{N}_{2}$ derived from (70) can be used. Moreover, in this region $\left|\tilde{N}_{1}\right|$ decreases with $z$. In fact, the first term of $(70)$ suggests that $\tilde{N}_{1}$ contributes with a term of order $z^{-3}$. So, up to this order, we obtain,

$$
N=\frac{4 \pi}{9}\left(\bar{\mu} z^{2}+\frac{\bar{\mu}^{3}}{\pi^{2}}\right)-\frac{2}{3 \pi} \bar{\mu}+\frac{7 \zeta(3)}{126 \pi^{3} z^{2}} \bar{\mu}+O\left(z^{-3}\right),
$$

The first two terms in the right hand side of (71), proportional to $R^{3}$ and $R$ respectively, reproduce equation (27) in Ref. [5], where only the first finite size corrections to the free energy are taken into account. In the approximation leading to (71) we were able to get the next, $1 / R$, term.

Finally, the Gibbs free energy can be obtained by integrating the mean number,

$$
R(G(z, \bar{\mu})-F(z))=-\int_{0}^{\bar{\mu}} N\left(z, \bar{\mu}^{\prime}\right) d \bar{\mu}^{\prime},
$$

where $F(z)$ stands for the Hemholtz free energy of the system. In fact, $F(z)$ has been studied in Ref. [2], so the expression above completely determines $G(z, \bar{\mu})$.

If the fermionic number is fixed, in media, to $N$, one can obtain (numerically) the chemical potencial as a function of $z, \bar{\mu}(z, N)$. Replacing in (72) we get

$$
R G(z, \bar{\mu}(z, N))=-\left[\int_{0}^{\bar{\mu}(z, N)} d \bar{\mu} \prime N(z, \bar{\mu} \prime)-R F(z)\right]
$$

The numerical evaluation of (73) for $N=1 / 2$, in a wide range of values of $z$, is shown in Fig. 2.

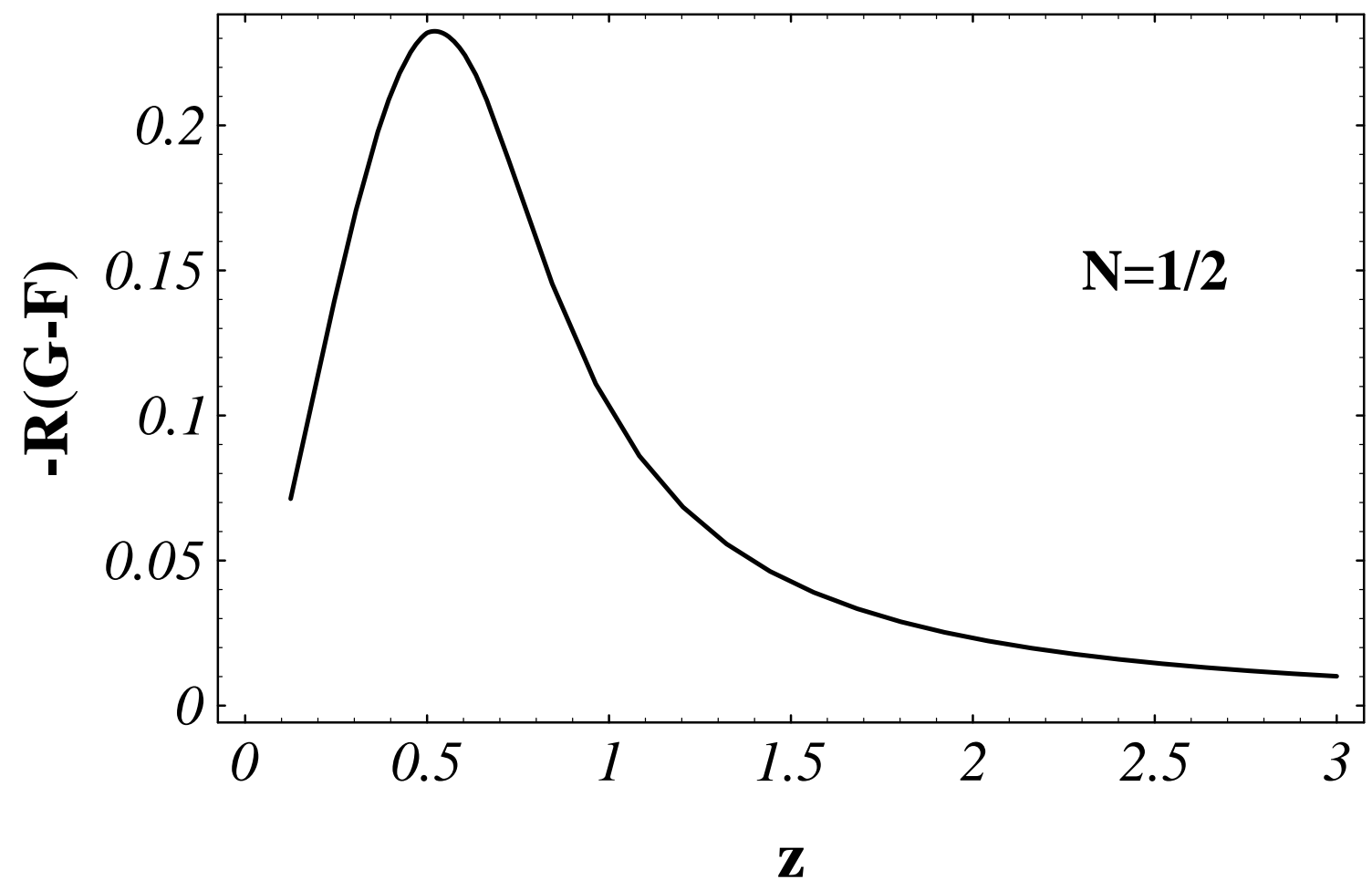

FIG. 2. Gibbs free energy at fixed fermionic number $N=1 / 2$ 


\section{Application to the model of quark-gluon droplet}

We will now apply our results to the study a simple model of hadrons: a static spherical droplet of confined quarks and gluons.

Considering the degeneracies of flavor, $N_{f}=2$, and color, $N_{c}=3$, we obtain, from (73)

$$
G_{\text {quarks }}=-\frac{N_{f} N_{c}}{R}\left[\int_{0}^{\bar{\mu}(z, N=3)} d \bar{\mu} \prime N(z, \bar{\mu} \prime)+z\left(-\beta F_{\text {fermions }}(z)\right)\right]
$$

where $-\beta F_{\text {fermions }}(z)$ is given in section III of Ref. [2].

On the other hand, in the 1-loop approximation, the gluon field can be described as 8 copies $\left(N_{g}=8\right)$ of the Abelian gauge field studied in Section IV of Ref. [2]. So,

$$
G_{\text {gluons }}=-\frac{N_{g}}{R} z\left(-\beta F_{\text {gauge }}\right)
$$

Finally, a bag stabilizing term, $B V$, is introduced. The bag constant is fixed to insure the pressure equilibrium when temperature reaches the critical value (which we take as $T_{0}=150 \mathrm{MeV}$ ). From the leading volume terms, we obtain

$$
B=\frac{37}{90} \pi T_{0}^{4}
$$

When the Casimir energies of quarks and gluons (obtained in Ref. [2] in the framework of an analytic regularization), which can be written as $C / R$ with an undetermined coefficient $C$, are not considered, the remaining part of the total Gibbs free energy,

$$
G(z, \bar{\mu}(z, N=3))=G_{\text {quarks }}+G_{\text {gluons }}+B V
$$

is as shown in Fig. 3 for different values of the temperature.

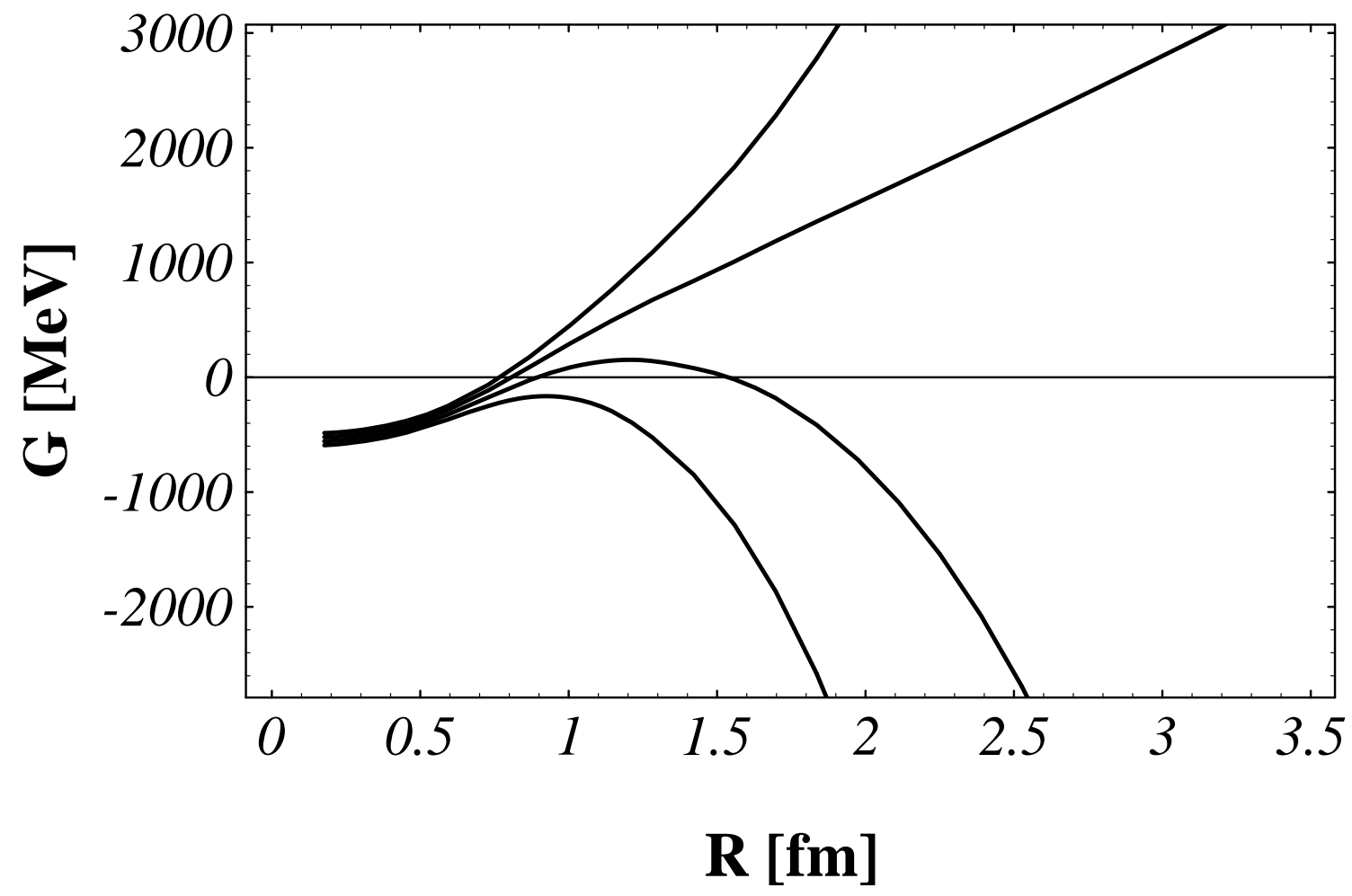

FIG. 3. Gibbs free energy for the quark-gluon droplet. From top to bottom: $T=140,150,160,170 \mathrm{MeV}$ 
The situation we find is consistent with the first order transition of a hadronic bag to a deconfined quark-gluon phase described in [5]: For each positive value of $C$, and for $0 \leq T<T_{0}$, an absolute minimum of $G(z, \bar{\mu}(z, N=3))$ appears at a finite value of $R$, corresponding to a stable bag configuration. When the temperature reaches $T_{0}$, this becomes only a local minimum representing a metastable state. When $T$ grows up, the local minimum and the local maximum approach each other. Finally, there is a critical temperature $T_{c}>T_{0}$ such that for $T>T_{c}$ there is no local minima at finite $R$, and only the deconfined phase can exist.

The computational techniques employed in this paper can, in principle, also be applied to more refined models of hadrons, such as the hybrid chiral bag model [22, 14]. In Ref. [23 the behavior of the total energy at $T=0$ and $\mu=0$ has been analyzed, showing a remarkable independence on the bag radius, in agreement with the so called Cheshire Cat Principle [14]. This justifies the proposal in [15,16], of looking for a deconfining transition only at the temperature (and $\mu$ ) dependent part of the free energy.

The Helmholtz free energy $(\mu=0)$ of a chiral bag can be obtained from the results in [2,24]. The method developed in the present paper could be applied to study the $\mu$ dependent part of the Gibbs free energy for this system. Notice that, for this model, boundary conditions (which depend on the chiral angle) make the evaluation of the Green's

function more difficult, since there symmetry corresponds to the diagonal subgroup of $S U(2)_{\text {rotational }} \otimes S U(2)_{\text {isospin }}$ (see Ref. [25] for the $T=0$ case). We will report on this subject elsewhere.

\section{ACKNOWLEDGEMENTS}

This work was supported in part by Fundación Andes - Antorchas under Contract No. C-12777-9, CONICET (Argentina), and FONDECyT (Chile) under Grant No. 1950797.

[1] H. Falomir et al., Journal of Mathematical Physics in press, (1995).

[2] M. D. Francia, Physical Review 50D, 2908 (1994).

[3] H. Falomir et al., funct-an/9411005 (unpublished).

[4] M. D. Francia, H. Falomir, M. Loewe, and E. M. Santangelo, Physical Review D51, 7132 (1995).

[5] M. G. Mustafa and A. Ansari, Zeitschrift für Physik C57, 51 (1993).

[6] E. V. Shuryak, The QCD Vacuum, Hadrons and Superdense Matter, Lectures Notes in Physics, Vol. 8 (World Scientific Publishing Co., Singapore, 1988).

[7] I. Tserruya, in Proceedings of the International Europhysics Conference on High Energy Physics (World Scientific Publishing Co., Brussels, 1995, 1996).

[8] A. Smilga, hep-ph/9604367 (unpublished).

[9] F. Karsch, in QCD 20 years latter, edited by P. M. Zerwas and H. A. Kastrup (World Scientific Publishing Co., Aachen, 1992, 1993).

[10] A. Barducci et al., Phys.Rev. D46, 2203 (1992).

[11] H. Leutwyler, in QCD 20 years latter, edited by P. M. Zerwas and H. A. Kastrup (World Scientific Publishing Co., Aachen, 1992, 1993).

[12] C. A. Dominguez, M. Loewe, and J. S. Rozowsky, Phys.Lett. B335, 506 (1994).

[13] K. J. Eskola and K. Kajantie, Zeitschrift für Physik C44, 347 (1989).

[14] M. Rho, Physics Reports 240, 1 (1994).

[15] H. Falomir, M. Loewe, and J. C. Rojas, Physics Letters 300, 278 (1993).

[16] M. Loewe and S. Perez-Oyarzún, Physics Letters 322B, 413 (1994).

[17] I. Mardor and B. Svetitsky, Physical Review 44D, 878 (1991).

[18] J. Cleymans, R. V. Gavai, and E. Suhonen, Physics Reports 130, 217 (1986).

[19] T. H. Hansson and R. L. Jaffe, Physical Review 28D, 882 (1983).

[20] M. Abramowitz and I. Stegun, Handbook of Mathematical Functions (Dover Publications, New York, 1970).

[21] P. J. Mulders, Physical Review D30, 1073 (1984).

[22] G. E. Brown, A. D. Jackson, M. Rho, and V. Vento, Physics Letters 140B, 285 (1984).

[23] M. D. Francia, H. Falomir, and E. M. Santangelo, Physics Letters B 371, 285 (1996).

[24] M. D. Francia, H. Falomir, and E. M. Santangelo, Physical Review D45, 2129 (1992).

[25] I. Zahed, A. Wirzba, and U.-G. Meissner, Annals of Physics 165, 406 (1985). 